\title{
Öffentliche Kulturförderung und Künstlersozialabgabepflicht
}

\author{
Landessozialgericht Berlin-Brandenburg, Urteil vom 15. Juli 2011 - L 1 KR 370/09
}

Eine Senatsverwaltung, die Stipendien an bildende Künstler vergibt und so diesen ermöglicht, ihre Werke im Rahmen einer Ausstellung zu präsentieren, ist nicht grundsätzlich nach § 24 KSVG abgabepflichtig.

\section{Tenor}

- Der Gerichtsbescheid des Sozialgerichts Berlin vom 3. Dezember 2009 und der Bescheid der Beklagten vom 15. November 2005 in der Gestalt des Widerspruchsbescheides vom 20. März 2006 werden aufgehoben.

Die Beklagte trägt die Kosten des Verfahrens.

Die Revision wird zugelassen.

\section{Tatbestand}

Im Streit steht ein Bescheid der Beklagten [Künstlersozialkasse bei der Unfallkasse des Bundes], wonach der Kläger, der als Senatsverwaltung für Wissenschaft, Forschung und Kultur, einen Galerieraum unter dem Namen "KunstBank" betrieb, insoweit dem Grunde nach abgabenpflichtig nach dem Künstlersozialversicherungsgesetz (KSVG) ist.

Die Senatsverwaltung vergab jährlich Arbeitsstipendien im Bereich der zeitgenössischen bildenden Kunst an in Berlin lebende und arbeitende Bildende Künstler. Die 20 Stipendiatinnen und Stipendiaten hatten die Möglichkeit, sich im Rahmen des Stipendiums mit einer Ausstellung der interessierten Öffentlichkeit vorzustellen. Die Ausstellungen fanden im Erdgeschoss des Gebäudes der Senatsverwaltung statt, der KunstBank. Es handelte sich nicht um Verkaufsausstellungen. Die Stipendiaten erhielten keine Honorare, lediglich eine (nicht pauschale) Materialaufwandsentschädigung. Eintrittsgelder wurden nicht erhoben.

Der Internetauftritt hierzu wurde von einem Mitarbeiter der Senatsverwaltung erstellt.

Die Beklagte erfuhr im Rahmen einer Internetrecherche im Juni 2005 von der KunstBank. Sie schickte der Senatsverwaltung ihren einschlägigen Fragebogen zu, welchen diese ausgefüllt mit Schreiben vom 7. September 2005 zurücksandte.

Die Beklagte stellte mit Bescheid vom 15. November 2005 fest, dass die Senatsverwaltung ein nach § 24 KSVG abgabepflichtiges Unternehmen betreibe. Es sei § 24 Abs. 1 Satz $1 \mathrm{Nr}$. 6 KSVG einschlägig, weil sie als Unternehmen eine Galerie oder einen Kunsthandel betreibe.

Der Kläger erhob Widerspruch. Zur Begründung führte er aus, Träger der Ausstellungsreihe sei nicht er, sondern der $\mathrm{N}$ e.V., welcher am Vorabend jeder Ausstellungseröffnung im Rah- men seiner Künstlergespräche die Stipendiaten vorstelle. Das Land erteile keine Aufträge an selbstständige Künstler oder Publizisten. Es sei kein verkaufsorientiertes Unternehmen. Es nehme keine künstlerischen oder publizistischen Leistungen für seine Zwecke in Anspruch und erziele diesbezüglich keine Einnahmen. Die Verwertung bzw. Vermarktung der Werke der Stipendiaten erfolge ausschließlich durch diese selbst. Die Ausstellungsreihe werde nicht vom Land Berlin, sondern ausschließlich vom Sparkassen Kulturfond des Deutschen Sparkassen- und Giroverbandes finanziert.

Die KunstBank wurde nach einem Brand zum 1. Februar 2006 geschlossen.

Die Beklagte wies den Widerspruch mit Widerspruchsbescheid vom 20. März 2006 zurück. Dem Internetauftritt sei sehr wohl zu entnehmen, dass es sich um eine Galerie handele. Die KunstBank sei laut Internetauftritt Bestandteil der Berliner Galerieszene. Die Angaben im Internet widersprächen auch dem Einwand, der N-Verein sei Träger der Ausstellungsreihe.

Die Beklagte setzte ferner, indem sie einem Widerspruch des Klägers gegen einen vorangegangenen Abgabenfestsetzungsbescheid abhalf, mit Bescheid vom 22. März 2006 die Abgaben für die Jahre 2001 bis 2004 sowie Vorauszahlungen bis einschließlich Februar 2006 unter Vorbehalt auf 0 EUR fest.

Gegen den Widerspruchsbescheid vom 20. März 2006 hat sich die am 13. April 2006 beim Sozialgericht Berlin (SG) erhobene Klage gerichtet. Zur Begründung hat der Kläger ausgeführt, er sei bereits kein Unternehmer im Sinne des Gesetzes. Das Land Berlin sei weder nach § 4 Abs. 1 Allgemeines Zuständigkeitsgesetz Berlin und dem dazugehörigen Zuständigkeitskatalog (ZusKatAZG) Betreiber einer Galerie noch Kunsthändler.

Als Träger der KunstBank sei der N B K beauftragt worden. Das Land Berlin habe keine Kunstwerke veräußern wollen noch daran mitwirken. Es sei auch kein Unternehmen, dessen Zweck überwiegend darauf gerichtet sei, künstlerische Werke öffentlich darzubieten. Es habe keine vertraglichen Beziehungen zwischen dem Kläger und den Stipendiaten gegeben. Das Land habe weder Aufträge an selbstständige Künstler erteilt noch Leistungen selbstständiger Künstler in Anspruch genommen oder diese vermarktet. 
Eine Fortsetzung des Programms „KunstBank" sei nicht beabsichtigt. Die Arbeiten von zehn Stipendiaten aus dem Förderzeitraum 2005/2006 erfolge auf der Grundlage eines neuen Konzeptes ab August 2006 in der Stiftung berlinische Galerie Landesmuseum für moderne Kunst, Fotografie und Architektur.

Zwar unterlägen unter Berücksichtigung der Grundsatzentscheidung des BSG vom 21. August 1996 (3 RK 31/95 SozR 3-5425 $\S 24 \mathrm{Nr}$. 15) auch Gebietskörperschaften, insbesondere die Länder, der Künstlersozialabgabe wie private Unternehmen, wenn Leistungen durch den Abschluss von Werkverträgen etc. ausgetauscht bzw. künstlerische oder publizistische Leistungen in Anspruch genommen oder verwertet würden, oder wenn eine Vergütung gezahlt werde. Hingegen könne nicht von Künstlersozialabgabepflicht ausgegangen werden, wenn ausschließlich öffentliche Kulturförderung betrieben werde, insbesondere wenn die Mittel einem entsprechenden Haushaltstitel entnommen würden, wenn Mittel auf Antrag vergeben würden oder Rechts- und Verwaltungsvorschriften die Grundlage der Vergabe bildeten und kein Rechtsanspruch auf die Vergabe der Projektmittel bestehe. Werbung im Sinne des KSVG meine überdies die Absatzwerbung als Unterfall der Wirtschaftswerbung. Nach der Rechtsprechung des BSG läge Werbung und Öffentlichkeit vor, wenn es um die positive Darstellung eines Unternehmens in der Öffentlichkeit und seiner Leistungen zum Zwecke der Gewinnung von Kunden bzw. Mitglieder gehe (Bezugnahme auf BSG Urteil vom 20. April 1994 - 3/12 RK 66/92).

Das Land Berlin betreibe ferner nur eine eigene Öffentlichkeitsarbeit. Werbung für Stipendiaten als Dritte im Sinne des $\S 24$ Abs. 1 Satz 1 Nr. 7 KSVG erfolge nicht. Der Kläger hat sich weiter auf ein Urteil des Sozialgerichts Hamburg vom 24. Juni 2009 (S 2 KR 553/07) berufen.

Die Beklagte hat auf das Urteil des BSG vom 21. August 1996 verwiesen. Es erscheine ihr lebensfremd, dass es sich bei der KunstBank nicht um Verkaufsausstellungen und damit eine Galerie gehandelt haben solle. Der Kläger sei aber jedenfalls nach $\S 24$ Abs. 1 Satz 1 Nr. 3 KSVG ein "sonstiges Unternehmen“, dessen „wesentlicher Zweck" die Darbietung künstlerischer Leistungen sei. Ein wesentlicher Zweck sei immer dann gegeben, wenn der Unternehmenszweck aufgrund gesetzlicher Grundlage durchgeführt werde (Bezugnahme auf BSG, Urteil vom 4. März 2004 - B 3 KR 6/03).

Die Ausstellungsmöglichkeit in den Räumen der Senatsverwaltung habe jedenfalls auch dem Zweck gedient, es den Künstler zu ermöglichen, die ausgestellten Werke an Interessierte zu veräußern. Es sei lebensfremd, davon auszugehen, dass die jungen Künstler nicht an einer Veräußerung ihrer Werke interessiert gewesen seien. Durch das zur Verfügung Stellen des Galerieraumes fördere der Kläger die Herstellung von Kontakten zwischen Künstlern und Endabnehmern. Hierdurch erfolge auch eine Förderung des Verkaufs von Kunstwerken. Dies sei als Kunsthandel zu qualifizieren (Verweis auf BSG, Urteil vom 20. April 1994 - 3/12 RK 33/92).
Die Beklagte hat sich ergänzend auch auf das Urteil des BSG vom 31. August 2000 berufen, wonach ein Kunstverein Unternehmer im Sinne des $\S 24$ Abs. 1 Satz 1 Nr. 3, 2. Alternative KSVG sei.

Aus Art. 20 Abs. 2 Berliner Verfassung („Das Land schützt und fördert das kulturelle Leben") folge die Pflichtaufgabe des Klägers, so dass im jedem Fall die grundsätzliche Abgabepflicht nach $\S 24$ Abs. 1 Satz 1 Nr. 3 Alternative 2 KSVG bestehe.

Nach Nr. 3 der genannten Vorschrift seien mehrere wesentliche Zwecke möglich. Gemäß Nr. 17 Abs. 2 ZustKatAZG zählten die "Landesangelegenheiten der Kunst" zu den Aufgaben der Hauptverwaltung. Die Senatsverwaltung habe eine eigene Abteilung K (Kunst) mit einer Unterabteilung bzw. einem Referat KE (Stipendien und Projektförderung). Aufgaben dieser Einheit sei nach eigenen Angaben die Förderung im Bereich der bildenden Kunst, u. a. durch die Vergabe durch Arbeitsstipendien und Ausstellungsmöglichkeiten gewesen. Also hätten diese Tätigkeiten der Senatsverwaltung zu ihren gesetzlichen Pflichtaufgaben gezählt.

Die grundsätzliche Abgabepflicht ergebe sich auch aus § 24 Abs. 1 Satz 1 Nr. 7 KSVG. Der Kläger betreibe Öffentlichkeitsarbeit für die von ihm geförderten Künstler.

Das SG hat die Klage mit Gerichtsbescheid vom 3. Dezember 2009 abgewiesen. Die Klage sei zwar zulässig. Der Kläger habe ein Rechtsschutzbedürfnis auf Aufhebung des angefochtenen Feststellungsbescheides. Mit der festgestellten Abgabepflicht dem Grunde nach seien grundsätzliche Nebenpflichten verbunden, nämlich die Melde-, Aufzeichnungs-, Auskunfts- und Vorlagepflichten nach $\S 27$ bis 29 KSVG.

Die Klage sei jedoch unbegründet. Der Kläger habe, solange er die KunstBank betrieben habe, dem Grunde nach der Abgabenpflicht unterlegen. Diese folge aus $\S 24$ Abs. 1 Satz 1 Nr. 7 KSVG. Der Kläger habe Öffentlichkeitsarbeit für Dritte betrieben, nämlich für die beteiligten Stipendiaten. Es komme nach der Rechtsprechung des BSG nicht darauf an, ob der Auftrag zur Öffentlichkeitsarbeit von einem Dritten oder - wie hier - vom Unternehmen selbst stamme, hier als Teil eines vom Land getragenen Programms sei, für dass der Gesetzgeber Haushaltsmittel zur Verfügung stelle (Bezugnahme auf BSG SozR 3-5425 § 24 Nr. 15). Entscheidend sei, dass der Kläger mit der Möglichkeit der Ausstellung der Werke der Stipendiaten für diese Öffentlichkeitsarbeit betrieben habe und darüber hinaus hierfür im Internet geworben habe. Genau dies sei auch Zweck der KunstBank gewesen. Der Kläger sei auch Abgabeschuldner. Die KunstBank sei durch den Kläger (durch die damalige Senatsverwaltung) betrieben worden. Wörtlich habe es im Internet geheißen, "die KunstBank ist der Galerieraum der Senatsverwaltung für Wissenschaft, Forschung und Kultur". § 24 Abs. 1 Satz 1 Nr. 7 KSVG differenziere nicht zwischen Kulturförderung und sonstigen Unternehmen. Es komme auch nicht darauf an, ob letztendlich 
abgabepflichtige Entgelte im Sinne des $\S 25$ KSVG festgestellt werden könnten. Die hier streitige Entscheidung über die Abgabepflicht dem Grunde nach bleibe nämlich davon unberührt.

Gegen diesen Gerichtsbescheid richtet sich die Berufung des Klägers, mit welcher er sein Vorbringen wiederholt und vertieft.

Die KunstBank sei unter anderem auch kein Unternehmen nach § 24 Abs. 1 Satz 1 Nr. 7 KSVG gewesen. Werbung sei nach der Definition des BSG die "positive Darstellung des Unternehmens in der Öffentlichkeit (so genannte Imagepflege) und seiner Leistungen zum Zweck der Gewinnung von Kunden". Werbung werde also im betriebswirtschaftlichen Sinne verstanden. Es gehe nicht um Information, sondern um Beeinflussung der - potentiellen - Kunden. Das Land jedoch habe nicht den Stipendiaten Kunden zuführen wollen. Öffentlichkeitsarbeit sei nach der gesetzlichen Vorschrift ("oder“) etwas anderes als Werbung. Sie stelle nicht ein bestimmtes Produkt heraus, sondern wolle eine gute Reputation herstellen. Auch ein solches Interesse fehlte der Senatsverwaltung. Das Land habe mit der KunstBank keine Public Relation betrieben.

\section{Der Kläger beantragt,}

den Gerichtsbescheid des Sozialgerichts Berlin vom 3. Dezember 2009 und den Bescheid der Beklagten vom 15. November 2005 in der Gestalt des Widerspruchsbescheides vom 20. März 2006 aufzuheben.

Die Beklagte beantragt,

die Berufung zurückzuweisen.

Sie verteidigt die angegriffene Entscheidung. Durch die öffentliche Kulturförderung des Klägers seien erhebliche finanzielle Mittel zur Verfügung gestellt worden, u. a., um die Öffentlichkeit auf die Ergebnisse der Kulturförderung hinzuweisen. Damit sei nicht nur Werbung für die eigene Institution betrieben worden, sondern auch für die Stipendiaten und deren Werke. Der Kläger könne sich auch nicht mit Erfolg darauf berufen, die Künstlerabgabepflicht scheide aus, weil dies zur Verminderung der Kunstförderung oder deren Einstellung führen müsse. Das BSG habe bereits entschieden, dass der Gesetzgeber davon ausgehe, dass Aufwendungen für die soziale Sicherung der selbstständigen Künstler und Publizisten zwangsläufig zu den mit der Inanspruchnahme von Künstlern verbundenen Kosten zählten (Bezugnahme auf BSG Urteil vom 31. August 2000 aaO.).

Auf die von den Beteiligten eingereichten Schriftsätze wird ergänzend Bezug genommen. Der Verwaltungsvorgang der Beklagten sowie die vom Kläger eingereichten Unterlagen waren Gegenstand der Erörterung in der mündlichen Verhandlung.

\section{Entscheidungsgründe}

Berufung und Klage haben Erfolg.

Die Klage ist zulässig. Auf die zutreffenden Ausführungen im angegriffenen Gerichtsbescheid wird gemäß § 153 Abs. 2 Sozialgerichtsgesetz (SGG) verwiesen. Auch wenn es die KunstBank in der Form zur Zeit des Erlass des Bescheides nicht mehr gibt, besteht zwischen den Beteiligten Dissens über die grundsätzliche Abgabenverpflichtung nach dem KSVG, soweit der Kläger Stipendiaten die Möglichkeit einräumt, in öffentlichen Ausstellungen ihre Werke zu präsentieren und hierauf auch in geeigneter Form aufmerksam macht, auch wenn der konkrete Anlass für das Tätigwerden der Beklagten speziell die Präsentationen in der KunstBank gewesen ist.

Damit ist gleichzeitig der Streitgegenstand umrissen: Der Grundlagenbescheid richtet sich ausweislich seines Tenors und seiner Begründung nicht gegen den Kläger schlechthin als nach dem KSVG verpflichteter Unternehmer (etwa als Betreiber von Theatern oder Volkshochschulen), sondern lediglich in seiner etwaigen Unternehmerschaft als Senatsverwaltung - bzw. Senatskanzlei - und konkret in der Form des Ausstellungsbetreiber bzw. Werbetreibender, der (gegenständliche) Kunstwerke seiner Stipendiaten der Öffentlichkeit präsentiert.

Machte die Aufhebung des konkreten Bescheides keinen Sinn mehr, wäre überdies von einer Klageänderung in Form einer Feststellungsklage (Fortsetzungsfeststellungsklage, § 131 Abs. 1 Satz 3 SGG) auszugehen. Der Kläger hätte ein berechtigtes Interesse an der Feststellung, dass der Verwaltungsakt (dann) rechtswidrig gewesen wäre.

Die Klage ist begründet.

Der Bescheid der Beklagten ist rechtswidrig und verletzt den Kläger in seinen Rechten.

Er ist als (Mit-) Betreiber der KunstBank nicht grundsätzlich nach § 24 KSVG abgabenpflichtig gewesen.

Unternehmer im Sinne des § 24 KSVG sind alle natürlichen oder juristischen Personen, deren Tätigkeiten einem der in dieser Vorschrift genannten Zwecke dient. Es ist weder eine Gewinnerzielungsabsicht erforderlich, noch kommt es darauf an, ob aus eigenem wirtschaftlichen Interesse, oder aus altruistischen Motiven gehandelt wird. Auch staatliche Kunstförderung unterliegt der Künstlersozialabgabepflicht, wenn sie sich einer der im Gesetz aufgeführten Handlungsformen bedient (BSG Urteil vom 31. August 2000 juris Rn. 12 mit weiteren Nachweisen).

Nach der Entscheidung des BSG vom 20. April 1994 (3/12 RK $66 / 92$; juris Rn. 12) genügt es bei staatlichen Institutionen, dass eine Kunstverwertung im Zusammenhang mit der Erfüllung 
von Aufgaben steht, die aus Haushaltszuweisungen, aus Beiträgen oder aus anderen Einnahmen finanziert wird. Insoweit ist der Unternehmerbegriff hier dem Grunde nach zu bejahen.

Die Stipendiatenförderung in der Form der KunstBank lässt sich jedoch unter keines der in § 24 KSVG aufgezählten Unternehmen subsumieren:

Die KunstBank war keine Galerie nach § 24 Abs. 1 Satz 1 Nr. $6 \mathrm{KSVG}$.

Der Senat verweist hierzu nach § 153 Abs. 2 SGG zur Vermeidung von Wiederholungen auf die Ausführung des SG im angegriffenen Gerichtsbescheid.

Die Beklagte kann sich für Gegenteiliges nicht mit Erfolg auf die Entscheidung des BSG vom 20. April 1994 (3/12 RK 33/92) berufen. Maßgeblich für die dortige Annahme, der dort klagende Kunstverein sei als abgabenpflichtig anzusehen, war der Umstand, dass es sich nach Auffassung des BSG nicht um das Ermöglichen einer reinen Selbstvermarktung durch die Künstler gehandelt hatte. Diese hatten sich vielmehr einer vermittelten Tätigkeit des Kunstvereins bedient, waren also in dessen Organisation des Verkaufs eingebunden. Der Kunstverein erzielte zudem durch Verkaufsprovisionen Einnahmen.

Eine ähnliche Einbindung in die Organisation von Verkaufsgeschäften hat hier unstreitig nicht stattgefunden. Der Kläger präsentierte lediglich die Arbeitsergebnisse der Stipendiaten. Die Objekte wurden aber nicht zum Verkauf angeboten. Es konnte nur als Nebeneffekt ein Kontakt zwischen Künstler und potentiellem Erwerber entstehen, obgleich dies nicht Zweck der Präsentationen gewesen ist. Ein gewollter Zusammenhang mit etwaigen Verkäufen bestand nicht, insbesondere sollte das Land keine Provision oder ähnliches erhalten.

Der Kläger ist auch kein Unternehmen nach § 24 Abs. 1 Satz 1 Nr. 3 KSVG gewesen, dessen wesentlicher Zweck darauf gerichtet gewesen ist, für die Darbietung der künstlerischen Werke (hier die der Stipendiaten) zu sorgen.

Aus dem Urteil des BSG vom 31. August 2000 (B 3 KR 27/99 R - SozR 3-5425 § 24 Nr. 19) kann zwar gefolgert werden, dass die KunstBank auch bezweckte, künstlerische Werke darzubieten im Sinne des § 24 Abs. 1 Satz 1 Nr. 3 KSVG.

Das Ausstellen von Werken der Darstellenden Kunst ist nämlich auch eine Darbietung künstlerischer Werke im Sinne dieser Vorschrift.

Es fehlt jedoch am Erfordernis, dass diese Darbietung wesentlicher Zweck des Unternehmens sein muss:

Zum einen betreibt das Land Berlin die Ausstellung der Werke seiner Stipendiaten nicht als Pflichtaufgabe.
Zum anderen ist der spezielle Aspekt der Ausstellung der Werke der Stipendiaten im Rahmen der Kulturförderung des Landes Berlin ein nur untergeordneter:

„Wesentliche Zwecke" des Unternehmens nach § 24 Abs. 1 S. 1 Nr. 3 KSVG sind bei Behörden und anderen öffentlichen Einrichtungen anzunehmen, wenn die Aufgaben eine durch Gesetz verordnete Pflicht darstellen (BSG Urt. v. 04. März 2004 - B 3 KR 6/03R. juris Rn. 23).

Das Ausstellen der Werke der Kunststipendiaten ist jedoch keine gesetzliche Pflichtaufgabe der zuständigen Senatsverwaltung bzw. Senatskanzlei:

Der ZustKatAZG gibt keine Auskunft über gesetzliche Pflichtaufgaben. Er regelt lediglich die behördliche Zuständigkeit in der Verteilung zwischen Bezirksämtern, Sonderbehörden einerseits und der Hauptverwaltung (Senatsverwaltungen) andererseits.

Aus Art. 20 Abs. 2 der Verfassung von Berlin folgt lediglich der ganz allgemeine Auftrag der Kulturförderung.

Im Gegensatz hierzu waren in dem soeben angeführten Urteil des BSG der dort klagenden Landesmedienanstalt aufgrund detaillierter Bestimmungen des dortigen Landesgesetzes die Aufgaben zugewiesen worden, einen „Offenen Kanal“ zu betreiben, zur Medienforschung Aufträge an wissenschaftliche Autoren zu erteilen sowie Schriftenreihen herauszugeben.

Eine gesetzliche Vorschrift, wonach die damalige Senatsverwaltung (bzw. heute die Senatskanzlei) zwingend Stipendien zu vergeben hatte/hat und zudem den jungen Künstlern ermöglichen muss, ihre Werke öffentlich zeigen zu können, gibt es hingegen nicht.

Die Ausstellung der Werke der Stipendiaten war auch rein tatsächlich keine wesentliche Aufgabe:

Im Falle der Klage eines Kunstvereines, der ohne Verkaufsabsichten Gemälde ausstellte (BSG Urt. v. 31. August 2000 - B 3 KR 27/99 R) hat das BSG darauf abgestellt, dass es nach dessen Satzung zu seinen vorrangigen Zwecken gehört habe, solche Ausstellungen zu veranstalten.

Soweit es um die Förderung des Künstlernachwuchses geht, steht demgegenüber die Stipendienvergabe selbst im Vordergrund. Die Präsentation in der Öffentlichkeit ist nachrangig.

Der Kläger ist ferner kein Unternehmen, das Werbung oder Öffentlichkeitsarbeit im Sinne des § 24 Abs. 1 Satz 1 Nr. 7 KSVG für andere oder nach $\S 24$ Abs. 1 S. 2 KSVG für sich betreibt:

Weder die Senatsverwaltung allgemein noch die KunstBank im Besonderen, geschweige denn das Land Berlin als solches, sind eine Werbe- oder Public Relationsagentur. 
§ 24 Abs. 1 Satz 2 KSVG kann zwar weiter auch einschlägig sein, soweit es um Eigenwerbung bzw. die eigene Öffentlichkeitsarbeit einer öffentlichen Körperschaft geht (BSG Urt. v. 20. April 1994 - 3/12 RK 66/92; SozR 3-5425Nr. 6).

Weder die Ausstellungen noch die entsprechende Internetseite sind jedoch Werbung oder Public Relation für die Senatsverwaltung oder das Land Berlin allgemein gewesen.

Die Senatsverwaltung hat - anders als die Ersatzkasse im Fall der Klage, der dem Urteil des BSG vom 20. April 1994 (3/12 RK 66/92) zu Grunde gelegen hat - mit Hilfe der Ausstellungen keine Eigenwerbung betrieben. Öffentliche Kunstförderung dient nicht der Eigendarstellung. Der Kläger weist vielmehr zutreffend darauf hin, damit dem Auftrag aus Art. 20 Abs. 2 Berliner Verfassung zu dienen, dass kulturelle Leben zu schützen und zu fördern.

Der Annahme des SG, dass es auch ausreicht, dass für die Künstler selbst Werbung gemacht wurde, teilt der Senat nicht:

Nach der speziellen gesetzlichen Vorschrift ist Anknüpfungspunkt der Künstlersozialversicherung nicht die Werbung als solche, sondern das Betreiben eines Unternehmens der Werbung oder der Öffentlichkeitsarbeit für Dritte.

Solches hat das Land Berlin nicht unternommen.

Die Abgabenpflichtigkeit nach Nr. 7 des § 24 Abs. 1 Satz 1 KSVG trägt dem Umstand Rechnung, dass auch Werbeagenturen oftmals nicht Arbeitnehmer, sondern selbstständige Künstler und Publizisten (z. B. als Werbetexter oder Grafiker) einsetzen. Nur insoweit ist der Zweck des KSVG einschlägig.

Ganz grundsätzlich findet die Belastung der Vermarkter mit der Künstlersozialabgabe zur Finanzierung eines Teils der Kosten der Sozialversicherung selbstständiger Künstler und Publizisten ihre Rechtfertigung in dem besonderen kulturgeschichtlich gewachsenen Verhältnis zwischen selbstständigen Künstlern und Publizisten auf der einen, sowie den Vermarktern auf der anderen Seite. Dieses Verhältnis hat einen spezifischen Charakter, der über ein bloßes, wechselseitiges AufeinanderAngewiesen-Sein, wie es etwa zwischen Produzenten und Handel oder Erzeugern und Verbrauchern besteht, hinausgeht. Künstler und Publizisten erbringen unvertretbare, d.h. höchstpersönliche Leistungen, die in besonderer Weise der Vermarktung bedürfen, um ihr Publikum und also ihre Abnehmer zu finden. Dieses Verhältnis hat gewisse symbiotische Züge; es stellt einen kulturgeschichtlichen Sonderbereich dar, aus dem eine besondere Verantwortung der Vermarkter für die soziale Sicherung der - typischerweise wirtschaftlich schwächeren selbstständigen Künstler und Publizisten erwächst, ähnlich der der Arbeitgeber für ihre Arbeitnehmer (so weitgehend wörtlich BVerfG, Beschluss vom 8. April 1987 - 2 BvR 909/82 u. a. BVerfGE 75, 108 bis 165 juris Rn. 130).
Dass die Ausstellung gleichzeitig Werbung für die Stipendiaten gewesen ist, reicht für die zu fordernde symbiotische Beziehung nicht aus. Der Kläger hat die Stipendiaten nicht arbeitnehmerähnlich eingesetzt.

Eine einschränkende Auslegung des § 24 Abs. 1 Satz 1 Nr. 7 KSVG ergibt sich unabhängig hiervon auch aus dem Umstand, dass die Feststellung der grundsätzlichen Verpflichtung zur Zahlung einer Künstlerabgabe kein Selbstzweck ist. Es geht vielmehr (nur) darum, im nächsten Schritt konkrete Abgaben zu erheben. Soweit es jedoch um Werbung für die Künstler selbst geht, können keine Bestimmungsgrößen des $§ 25 \mathrm{KSVG}$ einschlägig sein:

Nach § 25 Abs. 1 Satz 1 KSVG sind Bemessungsgrundlage der Künstlersozialabgabe die Entgelte für die Werke, die ein nach $\S 24$ KSVG Verpflichteter im Rahmen der dort aufgeführten Tätigkeiten an selbstständige Künstler oder Publizisten zahlt. Bemessungsgrundlage sind ferner auch die Entgelte, die ein (nicht abgabepflichtiger) Dritter für die künstlerischen Werke zahlt, die für einen zur Abgabe Verpflichteten erbracht wurden (Satz 2 des genannten Absatzes).

Ein Entgelt nach § 25 Abs. 1 Satz 1, Abs. 2 Satz 1 KSVG hat der Kläger den Stipendiaten nicht gezahlt. Die ausgestellten Werke sind weder von ihm angekauft noch angemietet worden. Es besteht auch zwischen der Stipendiumsleistung als solcher und den im Einzelnen ausgestellten Werken kein kausaler Zusammenhang, der es rechtfertigen könnte, die Stipendiumsleistung als Entgelt anzusehen.

Die Stipendiaten haben ihre künstlerischen Werke auch nicht für das Land Berlin erbracht im Sinne des $\S 25$ Abs. 1 Satz 2 KSVG.

Die Kostenentscheidung folgt aus § 197 a SGG in Verbindung mit $\S 154$ Abs. 1 Verwaltungsgerichtsordnung.

Die Revision ist wegen grundsätzlicher Bedeutung zuzulassen, § 160 Abs. 2 Nr. 1 SGG. (Entscheidung von der Redaktion bearbeitet.) 